\title{
Atlas web turístico de la reserva de la biosfera del Archipiélago de San Andrés
}

Tourist web atlas of San Andres Archipelago's biosphere reserve

\section{Resumen}

En los últimos años los mapas en línea o mapas en la web se han convertido en una herramienta importante para la difusión de datos espaciales, especialmente en temas como el turismo, debido a que son más útiles y flexibles que los convencionales basados en las características intrínsecas que ellos poseen. Herramientas como los Sistemas de Información Geográfica (SIG) que enmarcan igualmente a los llamados SIG en línea (web- GIS o SIG -web), permiten desarrollar diferentes aplicaciones basadas en información espacial como la producción de los atlas web, dentro de un ambiente académico.

Este artículo presenta los resultados del proyecto de investigación Diseño de un atlas en linea para el Archipiélago de San Andrés y Providencia, cuyo objetivo principal se basó en el desarrollo conceptual y técnico del diseño de una interfaz dinámica e interactiva de un atlas turístico en línea, que permita de la difusión de información geográfica en la red y ayude a fomentar un sector turístico de Colombia como es el de San Andrés y Providencia.

Un diseño integral del atlas incluye considerar los diferentes modelos, determinar el estado del conocimiento, caracterizar las variables para el caso de estudio, adquirir los datos en el trabajo de campo, definir y actualizar la información que hará parte del atlas en línea, con lo que se desarrolla un procedimiento cartográfico de elaboración y publicación de mapas en la red.

\section{Palabras clave}

Cartografía en la red, sIG en línea, datos espaciales, atlas.

\begin{abstract}
In the latest years the web maps has been the most important tool for the dissemination of spatial data, specially in topics like tourism, because this type of maps are more useful than the conventional maps, based on their
\end{abstract}

1 Ingeniera Catastral y Geodesta. MsC. In Geoinformation Systems. (ITC de Holanda), Grupo nide. Irocha@udistrital.edu.co

2 Ingeniero Catastral y Geodesta. Magíster en Geofisica. (Universidad Nacional de Colombia), Grupo NIDE. acardenas@udistrital.edu.co 
properties and flexibility. Tools like GIS on line and web cartography provide the elements to create a web atlas in an academic environment, to be useful for dissemination and presentation of spatial data related to tourism.

This paper is a result of the research project called "Diseño de un atlas en línea para un sector turístico de Colombia. Caso de estudio San Andrés". The aim of this project was to make a web atlas of the Archipelago of San Andres, Providencia and Santa Catalina, which contributes to increase the number of visitors to the islands, using a methodology that integrates tools like Geographic Information Systems and Information technology.

The web atlas design includes: the design of different models, to determine the state of art and knowledge about this topic, data acquisition, filed work on site, and to develop a model for a web map production line, based on international standards and applying concepts of GIS on line.

\section{Key Words}

Web Atlases, Grs on line, Web Cartography, Tourism, spatial data

\section{Introducción}

La difusión de los sitios turísticos en Colombia no es la más adecuada, debido a que la publicidad utilizada no resuelve las necesidades que demanda el mercado de hoy, redundando en pérdidas económicas y atraso en el desarrollo local [4]. Teniendo en cuenta esta consideración, se realizó el proyecto de investigación cuyo objetivo fue diseñar un atlas turístico en línea para el departamento Archipiélago de San Andrés y Providencia, mediante la utilización de Sistemas de Información Geográfica (sig) y tecnologías de la Información. La importancia del proyecto radica en los beneficios socioeconómicos directos que se pueden obtener si se tiene en cuenta que la econo- mía del Archipiélago está basada principalmente en el turismo, el comercio, la pesca y la agricultura en menor escala.

Dadas sus características particulares, el Archipiélago es una región insular con alto potencial turístico pero también alta vulnerabilidad ecológica, que requiere de políticas de desarrollo acordes con las necesidades de la región. Entre otros problemas detectados por diferentes investigadores del caribe colombiano, se pueden mencionar: la escasa movilización del turismo nacional e internacional, las oportunidades para un comercio libre, el desaprovechamiento de un potencial ecoturístico y la falta de posicionamiento como destino turístico por la inadecuada difusión de las cualidades del Archipiélago. [4].

El objetivo general del proyecto de investigación fue elaborar un atlas turístico mediante una metodología que integrara los conceptos de los Sistemas de Información Geográfica (SIG) y las herramientas cartográficas de visualización en Internet, para hacer más eficiente la promoción del sector turístico del país, a través de la red (www). Los siG se definen como el conjunto de herramientas que permiten capturar, almacenar, analizar, modelar y presentar información geográfica para la toma de decisiones. [1]

Los objetivos específicos del proyecto fueron contribuir al desarrollo sostenible del Archipiélago de San Andrés, mediante la difusión de datos espaciales del sector turístico en la red y definir los principales elementos para la producción de mapas turísticos en la red.

La investigación se realizó con el apoyo de la Gobernación de San Andrés y Providencia y la oficina de turismo, entidad interesada en utilizar el aplicativo desarrollado por la Universidad Distrital .

Los resultados obtenidos del proyecto mostraron que el atlas contribuye al desarrollo sostenible del 
Archipiélago de San Andrés, ya que su finalidad es promocionar el turismo en la isla -que incluye el ecoturismo- enmarcado en las políticas ambientales del Archipiélago, definidas por la Gobernación y CoRAlina (Corporación Autónoma Regional del Archipiélago), considerando que dichas políticas son estrictas en términos del uso de los recursos y conservación de los recursos naturales vulnerables.

\section{Área de estudio}

El Archipiélago de San Andrés, Providencia y Santa Catalina está conformado por las islas de San Andrés, Providencia y Santa Catalina, y un grupo de islas menores, atolones y bancos coralinos, que emergen sobre el Mar Caribe. Se encuentra localizado a 500 millas de Cartagena. Las tres islas principales ocupan una superficie continental de $49 \mathrm{Km}^{2}$, de los cuales 27 $\mathrm{Km}^{2}$ corresponden a la isla de San Andrés, $22 \mathrm{Km}^{2}$ a Providencia, $1 \mathrm{Km}^{2}$ a Santa Catalina y una extensión de mar territorial de $349.800 \mathrm{Km}^{2}$, ocupando aproximadamente el $10 \%$ del área total del caribe. Cuenta con uno de los arrecifes de barrera más extensos y productivos del Atlántico y del país.

El Archipiélago se encuentra ubicado al Noroeste del país, localizado a $12^{\circ} 32^{\prime} 51^{\prime \prime}$ de latitud norte y $81^{\circ}$ $43^{\prime} 03^{\prime \prime}$ de longitud oeste.

El Archipiélago isla de San Andrés, Providencia y Santa Catalina fue declarado Reserva de la Biosfera Seaflower declarada en el año 2000 por la UNESCO, como medida para conservar la biodiversidad y permitir el funcionamiento de los ecosistemas marinos y costeros, promover el uso sostenible de las prácticas amigables con el medio ambiente. Seaflower comprende extensos y variados ecosistemas representativos de la región, entre ellos se destacan arrecifes y parches de coral, atolones, praderas marinas, manglares y bosque tropical de transición. Los ambientes marinos costeros alrededor de la islas incluyen playas arenosas, bosques de manglar, ciénagas pequeñas, acantilados rocosos, charcos salinos, praderas de fanerógamas, arrecifes rocosos y de coral, fondos arenosos y lagunas arréciales de poca profundidad.

\section{Metodología}

La metodología utilizada se puede resumir en el desarrollo de las siguientes actividades:

\section{Diagnóstico:}

Se elaboró el diagnóstico de la información existente en cuanto a fotografías aéreas y turísticas, imágenes de satélite, mapas e información alfanumérica o temática sobre la isla de San Andrés en diferentes instituciones. Igualmente, se realizó una investigación sobre otras fuentes de información como son las imágenes de satélite, y se encontró que existían imágenes IKONOS del año 2003, con buena resolución para el desarrollo del proyecto. Una vez realizado el análisis de las fuentes de información existentes, se determinó que se debía adquirir el mapa topográfico escala 1:5000, las imágenes de satélite de San Andrés y Providencia y desarrollar el trabajo de campo para la toma de fotografías de los sitios turísticos de la isla.

\section{Adquisición y edición de los datos:}

Los datos espaciales y temáticos utilizados en el proyecto de investigación fueron proporcionados por la Gobernación del Archipiélago. La labor realizada por el grupo de investigación fúe editar y crear la topología de los datos de tal forma que pudieran ser utilizados en la aplicación desarrollada. Los datos espaciales incorporados se tomaron del mapa topográfico de la isla-escala 1:2000- elaborado para el desarrollo del Plan de Ordenamiento Territorial.

Para garantizar la estandarización cartográfica según las normas internacionales se utilizó el Datum wGS84 y la Proyección Universal Transversa de Mercator, zonas 17 y 18 . Meridiano Central $81^{\circ}$ al occidente y factor de escala 0.9996 , con las respectivas transformaciones entre Datums y la transformación de las 
coordenadas planas, se llevaron los datos geográficos al sistema de coordenadas mencionados.

Igualmente se editaron las curvas de nivel y se generaron modelos digitales de elevación de las superficies emergidas y de la topografía de la corteza oceánica basados en mapas de batimetría y animaciones en tres dimensiones (3D), utilizando el método Krigging ordinario.

La imagen IKONOS georreferenciada de la isla de San Andrés se procesó para generar un archivo multibanda en formato img, a partir de cuatro archivos individuales correspondientes a las bandas rojas, verdes, azules e infrarrojo cercano, en formato .tif y se utilizó para actualizar la información digital del Archipiélago.

\section{Trabajo de campo}

Uno de los instrumentos utilizados para la recolección de los datos fueron las dos visitas a la isla de San Andrés que realizó el grupo de investigación. En la primera salida de campo se realizó el reconocimiento directamente en la zona de estudio para actualizar el mapa censal análogo escala 1:5000. En este mapa se incorporaron básicamente los sitios turísticos representativos de la isla: hoteles, balnearios, playas, atractivos naturales, paisajes acuáticos, entre otros y su respectiva descripción física. Igualmente, se tomaron fotografías de los principales sitios turísticos y se elaboró un video para ser editado y colocado en la aplicación.

Teniendo en cuenta que al realizar la primera versión del atlas algunos sitios de interés no tenían la información requerida en la base de datos espacial, se realizó una segunda visita de campo donde se recogieron los datos y se tomaron las fotos de los sitios turísticos faltantes, especialmente del centro de la ciudad, y se completó el video de la isla.

\section{Diseño conceptualización y creación del atlas web}

La primera etapa en la conceptualización del atlas fue definir el significado para la investigación de un atlas turístico en línea. Para este proyecto se definió el atlas turístico en línea como: Un conjunto de mapas estáticos y dinámicos que presentan la información turística, de una forma interactiva y amigable para los diferentes usuarios que navegan en la red.

Los mapas estáticos son los más comunes en la red. El origen de estos mapas son productos cartográficos originales los cuales son rasterizados y puestos como mapas de celdas en la red. Los mapas dinámicos, por otra parte, funcionan bajo el concepto de animación, pueden ser de solo vista, que corresponden a modificaciones gráficas que ocurren en el tiempo en donde la animación corresponde a un estado visual dinámico que involucra cambios en su presentación, o interactivos, que permiten la intervención del usuario en el desarrollo normal de la presentación del mapa en el tiempo, por medio de herramientas que detienen, adelantan, adquieren escenas específicas de la reproducción de la animación, entre otras.

\section{Dentro del diseño del atlas se consideraron las siguientes actividades:}

a. Definición del contenido temático: Determina la estructura temática del atlas basado en los conceptos de los SIG en línea. El atlas turístico incluye el siguiente contenido: datos espaciales representados en mapas dinámicos y estáticos, fotos de los principales sitios turísticos, Información temática con descripción de los sitios turísticos, tablas con información estadística o temática, imágenes de satélite, videos de San Andrés y Providencia en tres dimensiones.

b. Estructura sintáctica: La estructura sintáctica incorpora la definición de los objetos espaciales y su organización jerárquica dentro del atlas. Para esto se elaboró el Catálogo de objetos turístico el 
cual presenta los objetos, su definición, clasificación y codificación para luego ser incorporados a la base de datos espacial. La clasificación y codificación de los objetos se caracteriza por estar dividido en temas, grupos y objetos.

c. Definición del lenguaje cartográfico: En esta etapa se define el empleo del lenguaje cartográfico mediante los símbolos a utilizar de acuerdo al Catálogo de símbolos.

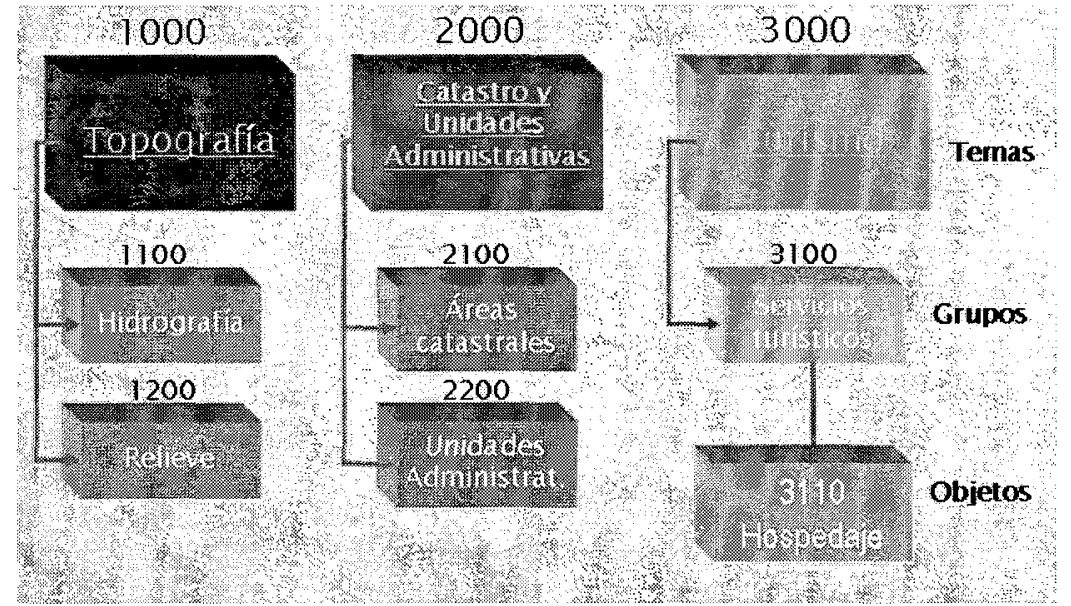

Figura No. 1: Modelo utilizado para el Catálogo de objetos
Los símbolos para este atlas turístico se basaron en diferentes fuentes gráficas prediseñadas, las cuales se aplicaron a los diferentes objetos turísticos.

d. Diseño cartográfico y publicación: En esta etapa se definen elementos como: cantidad de mapas, textos, fotos, formatos utilizados, escala y leyenda, para diseñar el visor de los mapas en Internet.

El atlas web está basado en el mapa turístico dinámico e interactivo de la isla, el cual presenta las siguientes características:

- El usuario puede visualizar la información a diferentes escalas de despliegue, partir de una escala pequeña (1:100.000) hasta una escala detallada (1:3000), de forma interactiva.

- Está publicado en Internet a través del servidor de mapas y puede ser consultado, mediante un navegador como Internet Explorer o Netscape Navegator.

Los datos se pueden consultar por medio de una aplicación previamente parametrizada que visualiza el sitio solicitado ubicándolo espacialmente en el mapa, y por medio de una foto del lugar con su respectiva descripción física.

\section{Arquitectura del atlas}

La arquitectura del atlas consiste de un servidor web, un servidor de aplicaciones, y un servidor de datos espaciales.

El servidor web es el encargado de recibir las peticiones de los clientes y enviar las paginas web publicadas hacia ellos, como resultado de las consultas dentro del atlas.

El servidor de aplicaciones ejecuta el proceso principal y maneja la distribución de carga de las consultas de los clientes un vez son recibidas. De acuerdo a las consultas enviadas, este remite dichas consultas al servidor espacial apropiado.

Una vez que los clientes acceden al atlas se ejecutan las consultas y como respuesta a dichas consultas, el usuario visualiza la información en formatos HTML.

\section{Publicación de los datos espaciales en la red}

Para publicar el mapa en la red se debe utilizar un servidor de mapas que posea las herramientas necesarias para la visualización de información espacial. Igualmente, se debe tener un navegador de Internet para que el usuario ejecute las acciones sobre la aplicación, en especial, cuando es un mapa dinámico interactivo. 
Desde un navegador (Internet Explorer o Netscape Navegator) el cliente ejecuta una acción, como por ejemplo un acercamiento en un área determinada del mapa, entonces el código se ejecuta en el servidor, el cual hace una traducción a peticiones mediante un conector, en el que se pide generar un nuevo mapa.

El servidor de aplicaciones consulta los datos y se genera una nueva imagen (en este caso .PNG) que se envía de vuelta al cliente.

\section{Resultados}

\section{Atlas turístico en la red}

El principal producto del proyecto de investigación es el atlas turístico en la red; el aplicativo se encuentra en la Intranet de la Universidad Distrital en el sitio: http://investigacion/ SanAndres/. El atlas tiene como propiedad que presenta la información espacial representada en mapas dinámicos y estáticos de los diferentes sitios turísticos del Archipiélago. Asociada a esta información existe una base de datos con las características de los sitios y una fotografía de cada lugar. Este atlas cumple con las características definidas durante su diseño, es decir es herramienta de consulta que muestra no sólo la información espacial en un mapa, sino que proporciona a la vez una fotografía y una descripción física del lugar solicitado por el usuario. Como ejemplo, se puede ver una consulta sobre un sitio turístico.

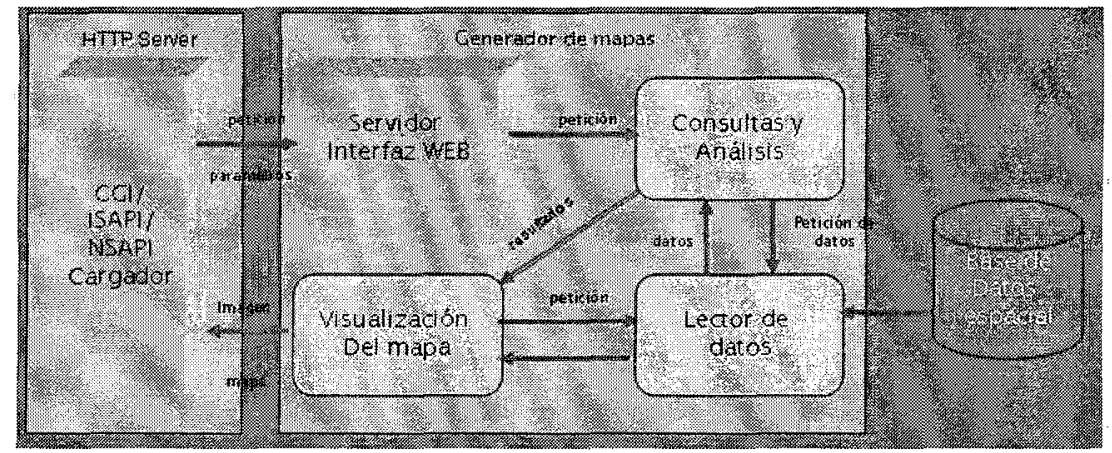

Figura No. 2: Esquema para publicación de mapas en la red

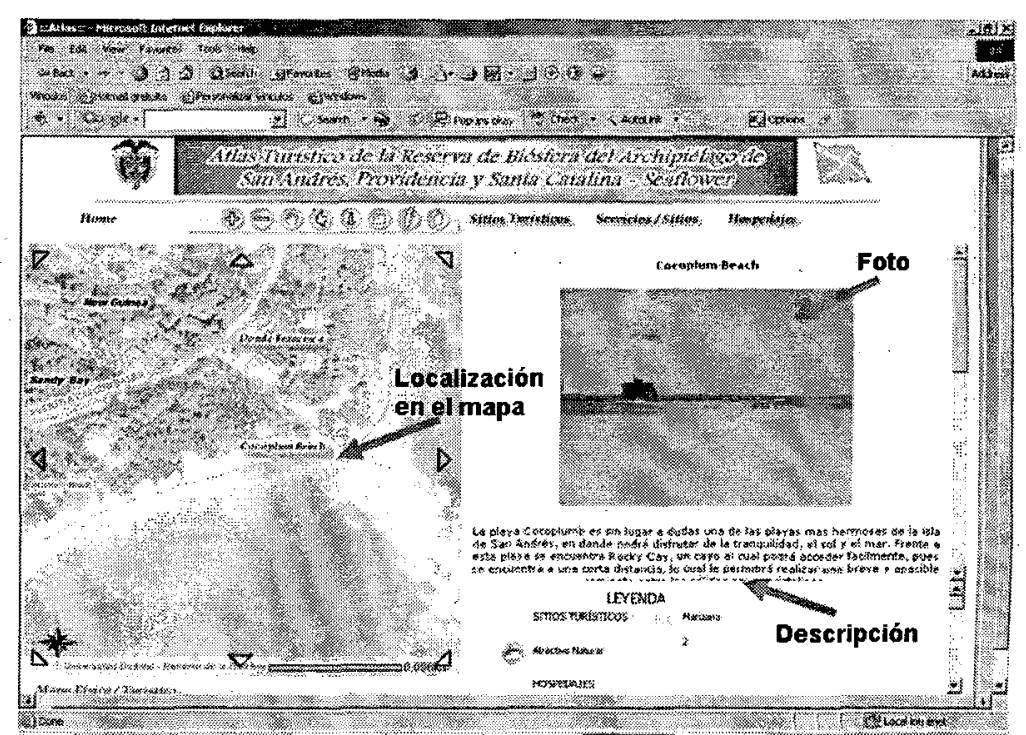

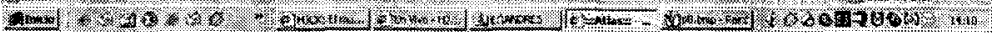
Figura No. 3: Bûsqueda de sitios turisticos

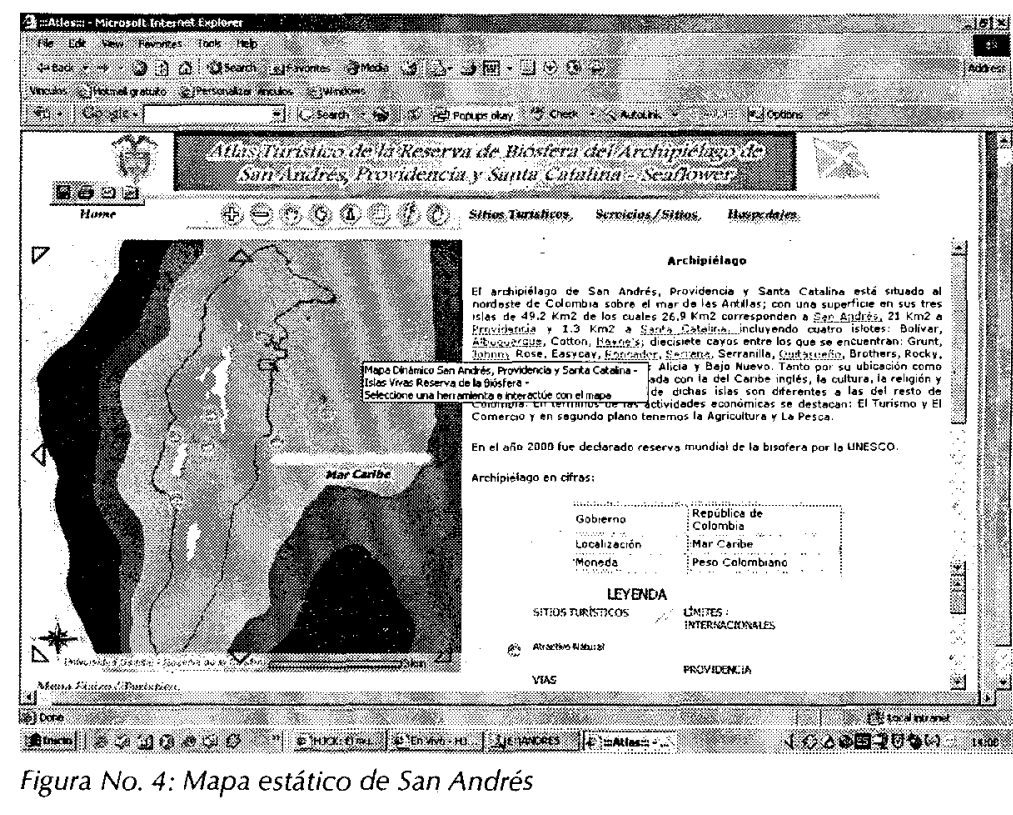




\section{Mapas web}

El atlas contiene varios tipos de mapas: estáticos, dinámicos y en 3 dimensiones. Algunos ejemplos relevantes son:

a. Mapa estático de todo el Archipiélago.

b. Mapa dinámico interactivo con imagen de satélite en el fondo, que muestra información desde una escala pequeña $(1: 100.000)$ hasta una escala grande (1:3000).

c. Modelo digital de elevación con animación

Catálogo de objetos y de símbolos turístico

El Catálogo de objetos es el documento que almacena la información de los objetos que se almacenaron en la base de datos espacial. Para este proyecto el Catálogo de objetos relaciona la información requerida para la producción del mapa turístico dinámico interactivo, lo que indica que la mayoría de los objetos son aquellos vinculados directamente con el tema del turismo.

El Catálogo de símbolos como su nombre lo indica relaciona la representación gráfica para cada uno de los objetos, relacionados en el catálogo de objetos. Los símbolos utilizados en este proyecto fueron fuentes de símbolos turísticos prediseñados tomados desde Internet.

\section{Línea de producción para mapas en la red}

Como resultado del trabajo realizado en la elaboración de los diferentes mapas que conforman el atlas, se desarrolló la línea de producción para generar mapas en la red, la cual no estaba prevista como un producto del proyecto de investigación, razón por la cual no fue contemplada en ninguna de las actividades preliminares.

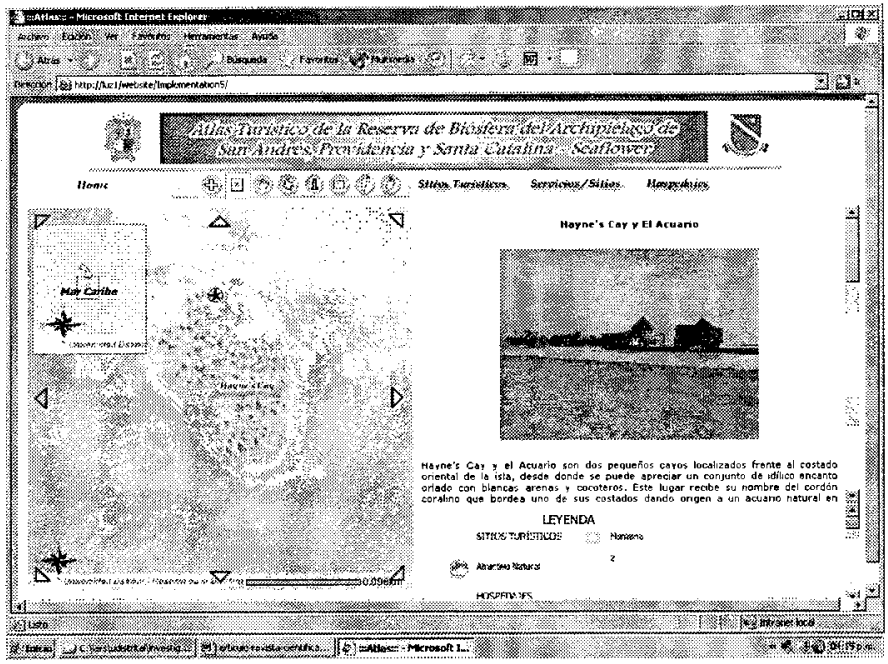

Figura No. 5: Mapa dinámico interactivo

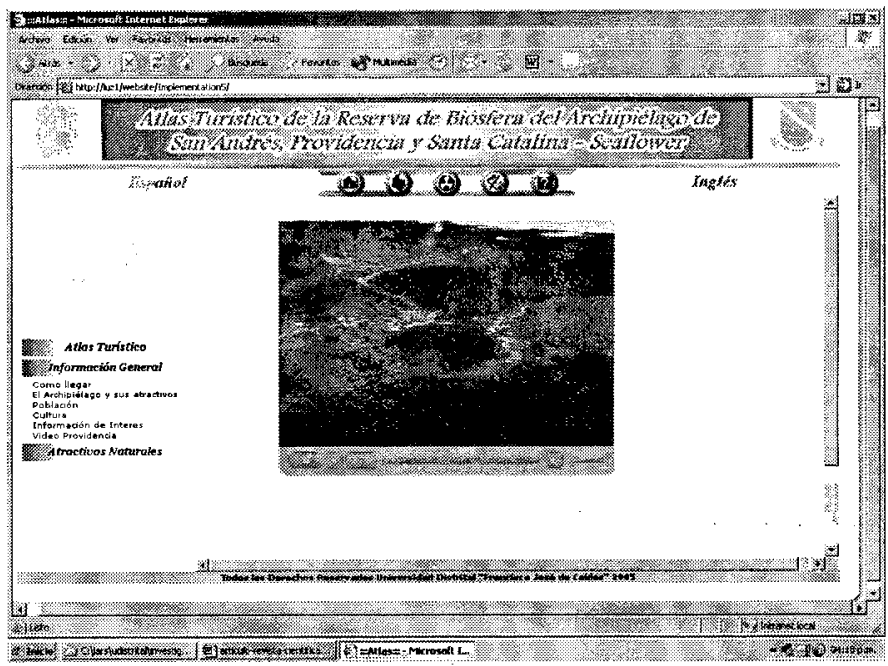

Figura No. 6: Modelo digital de elevación de Providencia

Esta línea de producción es una guía que relaciona las actividades básicas y un procedimiento general que sirve como herramienta y como soporte para que los productores realicen de una forma más técnica y sencilla los mapas que se publicarán en Internet.

Las actividades que conforman la línea de producción de mapas en la red ' son:

- Definición de elementos y componentes básicos del mapa 
- Definición, codificación y clasificación de los objetos del mapa

- Definición de la simbología

- Adquisición y preparación de los datos

- Creación del mapa web

- Creación de la interfaz web

- Publicación del mapa web

- Chequeo y ajuste

- Mantenimiento de los datos

\section{Conclusiones}

El atlas en línea del Archipiélago de San Andrés cumplió con el objetivo propuesto, proporcionando resultados adicionales como es la línea de producción de mapas dinámicos que fue desarrollada teniendo en cuenta la experiencia para elaborar los mapas dinámicos del atlas.

Publicar información Raster en la red como la imagen de Satélite IкONOS, tiene un grado de dificultad asociado con el tamaño del archivo y más aún cuando la información tiene una resolución espacial de $1 \mathrm{~m}$. Después de varias pruebas se pudo concluir el buen desempeño del atlas, teniendo en cuenta que el tiempo de acceso a la página principal es inferior a $4.5 \mathrm{seg}$., a la primera página de $1.2 \mathrm{seg}$. y a cualquier consulta de 0.35 seg. aproximadamente, comparado con otras aplicaciones similares en Internet.

El diseño del proyecto y de la base de datos permiten que se puedan implementar en otros casos, no sólo para el desarrollo turístico del país, sino con otros propósitos igualmente importantes, como la gestión de datos espaciales en la red.
En la publicación de datos espaciales en la red, es importante tener en cuenta los requerimientos de Hardware y Software, de esta manera se considera que en proyectos futuros es posible implementar este tipo de aplicaciones en software libre.

El aplicativo del atlas esta construido en una tecnología que adopta estándares internacionales establecidos por la oGC (Open Geospatial Consortium), esto garantiza que el servicio de mapas pueda llegar a ser consultado por diferentes tipos de usuarios a través de la red, es decir, aquellos que utilizarán el atlas a través de intranet o a los usuarios que eventualmente visitarán el sitio web para encontrar la información turística del lugar, o que navegando a través de la red encuentran el sitio y se detienen para tomar alguna información y posiblemente volver a visitarlo.

La línea de producción para generar mapas en la red se convierte en una herramienta importante para los productores de datos espaciales, teniendo en cuenta que no existe ningún tipo de documento que ayude a la creación técnica y especializada de este tipo de mapas en el país.

\section{Referencias y bibliografía}

[1] BURROUGH, P.; MCDONNELL R. (1998).Principles Of Geographical Information Systems: Spatial Information Systems And Geostatistics. Great Britain: Oxford University Press, 333 p.

[2] CHAMBERS, D. (1989). "Overview of GIS Database Design" ("Una mirada a el diseño de bases de datos para aplicaciones SIG") in GIS Trends, ARC News Spring, Redlands, California: Environmental Systems Research Institute.

[3] CORALINA. (2003). Archipiélago de San Andrés, Providencia y Santa Catalina: Una reserva de biosfera en el Caribe colombiano. 
[4] CORPORACIONES AUTONOMAS REGIONALES DE LA REGION CARIBE, (2005). Agenda para el desarrollo sostenible del Caribe colombiano.

[5] Plewe, B. (1997). GIS online. Information, retrieval, mapping, and the internet, s.l., Onword press.

[6] GOBERNACION DE SAN ANDRES (2003). Plantación departamental. Plan de Ordenamiento Territorial.

[7] GOODCHILD, M.F.; KEMP, K.K. NCGIA Core Curriculum in GIS. (Curso en SIG, del centro nacional de análisis geográfico espacial NCGIA), [Documento en línea]. U.S.A. National Center for Geographic Information and Analysis, University of California.

[8] KRAAK, M. J.; ORMELING, F. (1996). Cartography visualization of spatial dat.; s.l., Addison Wesley Longman.
[9] KRAAK, M.; BROWN. (2000). Web Cartography: developments and prospects. s.l., Taylor \& Francis.

[10] RINCÓN RIAÑO L.; GUTIÉRREZ MEDELLÍN R. (2004) Conceptualización e implementación del mapa dinámico en línea del departamento Archipiélago de San Andrés, Providencia y Santa Catalina. Bogotá, Universidad Distrital Francisco José de Caldas.

[11] ROCHA SALAMANCA L. y CÁRDENAS CONTRERAS A. (2006). Informe final del proyecto de investigación diseño de un atlas en línea para un sector turístico de Colombia. Caso de estudio San Andrés. Bogotá, Universidad Distrital Francisco José de Caldas.

[12] ROCHA SALAMANCA L. (2006). La linea de producción de mapas en la red (www), una herramienta útil para la difusión de datos espaciales. Memorias XII Simposio de Percepción Remota y SIG. SELPER. 